\section{EFFECTIVENESS OF THROMBOLYSIS WITH INTRAVENOUS ALTEPLASE FOR ACUTE ISCHEMIC STROKE IN OLDER ADULTS}

To the Editor: Thrombolysis with intravenous alteplase is widely accepted as an effective treatment for individuals with acute ischemic stroke, ${ }^{1}$ but few individuals aged 80 and older are treated with alteplase. ${ }^{2}$ The risk:benefit ratio of thrombolysis might be less favorable with older age because of a higher risk of adverse events and lower efficacy. ${ }^{3,4}$ Outcomes in older adults treated with alteplase has been studied mostly in comparison with younger individuals, ${ }^{4,5}$ but to reach a firm conclusion concerning the appropriateness of treating older adults with thrombolysis, one should also contrast treatment with no treatment. Individuals aged 80 and older with stroke have a higher casefatality rate, longer hospitalization, and are less likely to be discharged home than younger individuals. ${ }^{6}$ The results of a recent collaborative project suggest that individuals aged 80 and older derive similar benefit from treatment with intravenous alteplase as younger individuals. ${ }^{7}$ The aim of the present study was to assess the effectiveness of thrombolysis in relation to age in an unselected prospective observational cohort, in which all individuals with an ischemic stroke admitted within 4 hours of symptom onset were registered.

\section{PARTICIPANTS AND METHODS}

During a 2-year period from 2005 to 2007, all individuals aged 18 and older with acute ischemic stroke admitted within 4 hours of symptom onset were registered in 12 hospitals in the Netherlands. Participant data included demographics and baseline characteristics. All participants were followed up at 3 months after hospitalization by telephone, which they had consented to. A good outcome at 3 months was defined as a score on the modified Rankin Scale of 2 or less. The safety endpoints were death and symptomatic intracranial bleeding (sICH), defined as a hemorrhage confirmed using computed tomography scan and leading to a greater deficit on the National Institutes of Health Stroke Scale (NIHSS) than at baseline. A multivariable logistic regression model with adjustment for prognostic variables (age, sex, systolic blood pressure, NIHSS score at admission, diabetes mellitus, atrial fibrillation, history of previous stroke, and heart failure) was used to assess the effectiveness of thrombolysis according to age category.

\section{RESULTS}

Overall, 1,657 individuals were registered, of whom 446 were aged 80 and older and 1,211 were younger than 80 . Table 1 shows baseline characteristics, stroke severity, and outcome. The adjusted odds ratio (aOR) for good outcome was $1.19(95 \%$ confidence interval $(\mathrm{CI})=0.71-1.98)$ in the older age category and $1.48(95 \% \mathrm{CI}=1.08-2.04)$ in the younger age category. Heterogeneity between age strata was tested for by computing the Mantel-Haenszel ORs (MH-ORs) according to treatment, adjusted for NIHSS score at admission. The overall MH-OR for good outcome was $1.54(95 \% \mathrm{CI}=1.18-2.01)$. There was no

Table 1. Baseline Participant Characteristics and Outcomes According to Treatment and Age Group

\begin{tabular}{|c|c|c|c|c|}
\hline \multirow[b]{2}{*}{ Characteristic and Outcome } & \multicolumn{2}{|c|}{$\geq \mathbf{8 0}$} & \multicolumn{2}{|c|}{$<80$} \\
\hline & $\begin{array}{l}\text { No Thrombolysis, } \\
\qquad n=283\end{array}$ & $\begin{array}{l}\text { Thrombolysis, } \\
\qquad n=163\end{array}$ & $\begin{array}{l}\text { No Thrombolysis, } \\
\qquad n=678\end{array}$ & $\begin{array}{l}\text { Thrombolysis, } \\
n=533\end{array}$ \\
\hline \multicolumn{5}{|l|}{ Characteristic } \\
\hline Age, mean \pm SD & $85 \pm 4$ & $85 \pm 4$ & $66 \pm 11$ & $64 \pm 12$ \\
\hline Male, $\mathrm{n}(\%)$ & $120(42)$ & $70(43)$ & $400(59)$ & $312(59)$ \\
\hline Prior stroke or transient ischemic attack, $\mathrm{n}(\%)$ & $64(23)$ & $31(19)$ & $158(23)$ & $78(15)$ \\
\hline Myocardial infarction, $\mathrm{n}(\%)$ & $45(16)$ & $22(14)$ & $94(14)$ & $66(13)$ \\
\hline Peripheral artery disease, $\mathrm{n}(\%)$ & $39(14)$ & $14(9)$ & $65(10)$ & $40(8)$ \\
\hline Heart failure, $n(\%)$ & $35(12)$ & $22(14)$ & $44(7)$ & $31(6)$ \\
\hline Hypertension, n (\%) & $153(54)$ & $80(49)$ & $356(53)$ & $257(48)$ \\
\hline Diastolic blood pressure, $\mathrm{mmHg}$, mean $\pm \mathrm{SD}$ & $82 \pm 19$ & $81 \pm 17$ & $86 \pm 17$ & $84 \pm 15$ \\
\hline \multicolumn{5}{|l|}{ Outcome } \\
\hline $\begin{array}{l}\text { Good outcome (modified Rankin Scale score } \leq 2 \text { ), } \\
n(\%)\end{array}$ & $96(35)$ & $46(29)$ & $441(68)$ & $287(57)$ \\
\hline $\begin{array}{l}\text { Fully adjusted odds ratio for good outcome } \\
(95 \% \text { confidence interval })^{*}\end{array}$ & $1.19(0.71-1.98)$ & & $1.48(1.08-2.04)$ & \\
\hline $\begin{array}{l}\text { Symptomatic intracranial bleeding } \\
\text { complication, } \mathrm{n}(\%)\end{array}$ & - & $12(7.4)$ & - & $24(4.5)$ \\
\hline
\end{tabular}

$100 \%$ is the total participants registered within one age category per treatment arm.

"Multiple logistic regression model, including age, sex, atrial fibrillation, diabetes mellitus, previous stroke, and blood pressure at admission.

$\mathrm{SD}=$ standard deviation. 
evidence of heterogeneity of ORs between the age categories (test of homogeneity of ORs, $P=.39$ ). Participants aged 80 and older with stroke had a higher case-fatality rate and a higher rate of sICH than younger patients when treated with thrombolysis $(7.4 \%$ vs $4.5 \%$; relative risk $=1.51,95 \% \mathrm{CI}=0.77-2.97)$.

\section{DISCUSSION}

This retrospective analysis of unselected individuals suggests that thrombolysis for acute ischemic stroke leads to better outcomes than lack of treatment with alteplase in individuals aged 80 and older as well. These results confirm the observations in the Safe Implementation of Treatment in Stroke-International Stroke Thrombolysis Registry (SITS-ISTR). This is the largest prospective observational study of outcomes of thrombolysis in individuals aged 80 and older, with 21,242 participants, of whom 1,831 were aged 80 and older. The main finding in SITS-ISTR was that the overall rate of symptomatic and asymptomatic $\mathrm{ICH}$ was not greater in those aged 80 and older. ${ }^{8}$ The comparison of these data with those from matched historical controls showed that older age is associated with poorer outcomes, but the association between thrombolysis treatment and better outcomes is maintained in very elderly people. $^{7}$ The SITS-MOST registry is an uncontrolled study and a voluntary registry, so consecutive inclusion and absence of selection is difficult to guarantee. ${ }^{9}$ The Third International Stroke trial will provide stronger evidence; this randomized controlled trial seeks to determine whether a wider range of individuals may benefit from alteplase, including patients aged 80 and older. ${ }^{10}$ Additional evidence of a beneficial effect of thrombolysis is presented here because the stratified analysis shows a significant overall effect of thrombolysis, with no evidence of heterogeneity within the different age strata. This treatment should not be withheld from older adults, who should be treated directly, or when there is substantial doubt about the effect of treatment, these individuals should be asked to participate in randomized clinical trials.

Maaike Dirks, MD Peter J. Koudstaal, MD, PhD Diederik W. J. Dippel, MD, PhD Department of Neurology Erasmus MC University Hospital Rotterdam

Louis W. Niessen, MD, PhD Jeroen D. H. vanWijngaarden, $P h D$ Institute for Health Policy and Management Erasmus University Rotterdam Rotterdam, the Netherlands

Louis W. Niessen, MD, PhD School of Medicine, Health Policy and Practice University of East Anglia Norwich, UK

Louis W. Niessen, MD, PhD Department of International Health Johns Hopkins School of Public Health Baltimore, Maryland
Cees L. Franke, $M D, P h D$

Department of Neurology, Atrium Medical Center Parkstad, Heerlen

Robert J. van Oostenbrugge, $M D, P h D$ Department of Neurology Maastricht University Medical Center Maastricht, the Netherlands

\section{ACKNOWLEDGMENTS}

We thank all stroke care teams who participated in this study.

Conflict of Interest: The editor in chief has reviewed the conflict of interest checklist provided by the authors and has determined that the authors have no financial or any other kind of personal conflicts with this paper.

This work was supported by the Netherlands Organization for Health Research and Development (Grant 945-14-217), the national health council appointed by the Ministry of Health and the Netherlands Organization for Scientific Research to promote quality and innovation in the field of health.

Author Contributions: Maaike Dirks: Conception and design of the article, acquisition of data, analysis and interpretation of data, drafting of the article, and approval of the final version. Louis W. Niessen, Peter J. Koudstaal, Cees L. Franke, and Robert J. van Oostenbrugge: Conception and design of the article, critical revision of the article for important intellectual content, and approval of the final version. Jeroen van Wijngaarden: Acquisition of data, critical revision of the article for important intellectual content, and approval of the final version. Diederik W.J. Dippel: Conception and design of the article, analysis and interpretation of data, critical revision of the article for important intellectual content, and approval of the final version.

Sponsor's Role: The funder had no role in the design, methods, subject recruitment, data collections, analysis, or preparation of this paper.

\section{REFERENCES}

1. Wardlaw JM, Murray V, Berge E et al. Thrombolysis for acute ischaemic stroke. Cochrane Database Syst Rev 2009;CD000213.

2. Katzan IL, Hammer MD, Hixson ED et al. Utilization of intravenous tissue plasminogen activator for acute ischemic stroke. Arch Neurol 2004;61: $346-350$.

3. Larrue V, von Kummer R, Muller A et al. Risk factors for severe hemorrhagic transformation in ischemic stroke patients treated with recombinant tissue plasminogen activator: A secondary analysis of the EuropeanAustralasian Acute Stroke Study (ECASS II). Stroke 2001; 32:438-441.

4. Forster A, Szabo K, Kreisel S et al. Thrombolysis in very old people with stroke: Stroke subtypes, patterns, complications, and clinical outcome. J Am Geriatr Soc 2011;59:178-180.

5. Engelter ST, Reichhart M, Sekoranja L et al. Thrombolysis in stroke patients aged 80 years and older: Swiss survey of IV thrombolysis. Neurology 2005;65:1795-1798.

6. Saposnik G, Cote R, Phillips S et al. Stroke outcome in those over 80: A multicenter cohort study across Canada. Stroke 2008;39:2310-2317.

7. Mishra NK, Ahmed N, Andersen G et al. Thrombolysis in very elderly people: Controlled comparison of SITS International Stroke Thrombolysis Registry and Virtual International Stroke Trials Archive. BMJ 2010;341: c6046.

8. Ford GA, Ahmed N, Azevedo E et al. Intravenous alteplase for stroke in those older than 80 years old. stroke 2010;41:2568-2574. 
9. Wahlgren N, Ahmed N, Davalos A et al. Thrombolysis with alteplase for acute ischaemic stroke in the Safe Implementation of Thrombolysis in Stroke-Monitoring Study (SITS-MOST): An observational study. Lancet 2007;369:275-282.

10. Major ongoing stroke trials-Third International Stroke Trial (IST-3). Stroke 2005;36:e160-e161.

\section{DEPRESSIVE SYMPTOMS INCREASE THE RISK OF MORTALITY IN OLDER MEXICAN COMMUNITY-DWELLING ADULTS}

To the Editor: Depressive symptoms (DSs) are common in older adults, and their prevalence varies depending on the instrument used to measure them and the population studied. DSs represent a major public health concern because of their association with several adverse health outcomes such as greater use of medical services, institutionalization, functional and cognitive decline, and quality of life. Mortality, as an adverse outcome of DS, has been studied in the context of different diseases, but epidemiological reports from developing countries in this respect are scarce, so the main purpose of this 2-year longitudinal study of 2,615 persons was to determine whether DSs are an independent predictor of mortality in a sample of Mexican community-dwelling older adults without DSs at baseline.

Participants were adults aged 70 and older, all included in the Mexican Health and Aging Study, who were evaluated twice (2001 and 2003). DSs were established using a modified version of the Center for Epidemiologic Studies Depression Scale (CES-D), ${ }^{1}$ and time of death was obtained from interviews with the family or proxy relative at the 2-year follow-up. Cox proportional hazards models were performed to estimate the risk of death after adjusting for multiple covariates (age, sex, educational level, hypertension, diabetes mellitus, stroke, ischemic cardiopathy, visual impairment, urinary incontinence, cognitive impairment, smoking, alcohol use, and baseline activity of daily living (ADL) disability). The interaction terms between DSs and every one of all covariates were introduced into the final models, and their statistical significance was tested using backward selection. All statistical tests were performed at the .05 level.

\section{RESULTS}

Mean age was 76.4 (range: $70-105$ ); $52.9 \%$ were female. Hypertension $(42 \%)$ was the most frequent chronic disease. At baseline, 9.3\% had ADL disability. DSs were present in 1,127 (43.1\%) individuals. As expected, at baseline, participants with DSs were older, more likely to be female, and less educated and reported more chronic diseases. In addition, participants identified as having DSs reported more visual and hearing impairment and more ADL disability, and smoke and consumed alcohol less frequently than participants without DSs.

The incidence of death was $8.8 \%(\mathrm{n}=231)$ at the 2-year follow-up. Participants who had died during follow-up had reported more DSs at baseline than those who were still alive $(11.5 \%$ vs $6.8 \% ; P<.001)$. The unadjusted Cox proportional hazard model showed that presenting DSs at baseline almost doubled the risk of death at 2 years
Table 1. Cox Proportional Hazard Model of Death Estimates Over 2 Years of Follow-Up

\begin{tabular}{lcc}
\hline \multicolumn{1}{c}{ Adjustment } & $\begin{array}{c}\text { Hazard Ratio (95\% } \\
\text { Confidence Interval) }\end{array}$ & $\boldsymbol{P}$-Value \\
\hline Unadjusted & $1.74(1.34-2.25)$ & $<.001$ \\
Adjusted & $1.61(1.24-2.10)$ & $<.001$ \\
Age & $1.66(1.27-2.16)$ & $<.001$ \\
Sex & $1.68(1.29-2.19)$ & $<.001$ \\
Education level & $1.63(1.24-2.13)$ & $<.001$ \\
Hypertension & $1.62(1.23-2.12)$ & .001 \\
Diabetes mellitus & $1.61(1.23-2.11)$ & .001 \\
Stroke & $1.58(1.21-2.08)$ & .001 \\
Ischemic cardiopathy & $1.45(1.04-1.99)$ & .004 \\
Urinary incontinence & $1.32(1.10-1.92)$ & .009 \\
Visual impairment & $1.50(1.12-2.02)$ & .007 \\
Cognitive impairment & $1.50(1.11-2.01)$ & .007 \\
Smoking & $1.47(1.01-1.97)$ & .01 \\
\hline Alcohol intake & $1.36(1.01-1.83)$ & .04 \\
Disability in activities & & \\
\hline of daily living & & \\
\hline
\end{tabular}

(Table 1). DSs have also been proven to be a statistically significant predictor of death after adjusting for sociodemographic and health covariates because they increase its risk by $36 \%$.

\section{DISCUSSION}

The present study showed that DSs increase mortality risk regardless of multiple covariates, as has been found in other rural and urban populations (the majority Caucasians). ${ }^{2-6}$ Despite being a common concern in geriatrics, in Latin America, this association has been little explored. In Mexico, depression has been characterized according to its prevalence and sociodemographic characteristics and its effect on quality of life $e^{1,7}$ but not its relationship with mortality.

Diverse theories have been proposed to explain how DSs increase mortality (immunosuppression, hormonal changes, sympathetic tone increase, diminished vagal tone, and exacerbation of comorbidities), but the most plausible is that those with DSs are more likely to develop unhealthy behavior and habits, have poorer adherence to treatments, and use fewer healthcare services. Another hypothesis is that DSs generate more disability, although the mechanisms by which greater mortality might occur are unknown. ${ }^{6}$

Self-reported data and short follow-up could be the main limitations of the present study, and results must be interpreted carefully. In spite of the limits, the strength of this study is its population-based design, and to the knowledge of the authors, it is among the few in Latin America that explore this relationship. Given that depression is often unrecognized and undertreated in older adults, awareness of the potential for loss of life, as well as the potential for treatment, may help to improve this situation. Whatever the mechanism is, the effect of DSs on mortality seems to be evident, and screening for DS as part of the routine evaluation of older adults, especially in primary care, would be of great importance. 